\title{
Angular stripe phase in spin-orbital-angular-momentum coupled Bose condensates
}

\author{
Xiao-Long Chen $\odot,{ }^{1,2}$ Shi-Guo Peng, ${ }^{3, *}$ Peng Zou, ${ }^{4, \dagger}$ Xia-Ji Liu, ${ }^{2}$ and Hui $\mathrm{Hu}^{2}$ \\ ${ }^{1}$ Institute for Advanced Study, Tsinghua University, Beijing 100084, China \\ ${ }^{2}$ Centre for Quantum and Optical Science, Swinburne University of Technology, Melbourne, Victoria 3122, Australia \\ ${ }^{3}$ State Key Laboratory of Magnetic Resonance and Atomic and Molecular Physics, Wuhan Institute of Physics and Mathematics, \\ Chinese Academy of Sciences, Wuhan 430071, China \\ ${ }^{4}$ College of Physics, Qingdao University, Qingdao 266071, China
}

(Received 24 April 2020; accepted 30 June 2020; published 28 July 2020)

\begin{abstract}
We propose that a superfluid with supersolidlike properties, i.e., angular stripe phase, can be realized in a pancakelike spin-1/2 Bose gas with spin-orbital-angular-momentum coupling. We predict a rich ground-state phase diagram, including the vortex-antivortex pair phase, the half-skyrmion phase, and two different angular stripe phases. The stripe phases feature a modulated angular density-density correlation with a sizable contrast and can occupy a relatively large parameter space. The low-lying collective excitations, such as the dipole and breathing modes, show distinct behaviors in different phases. The existence of the stripe phase is also clearly indicated in the energetic and dynamic instabilities of collective modes near phase transitions. Our predictions of the angular stripe phase could be readily examined in current cold-atom experiments with ${ }^{87} \mathrm{Rb}$ and ${ }^{41} \mathrm{~K}$.
\end{abstract}

DOI: 10.1103/PhysRevResearch.2.033152

\section{INTRODUCTION}

Owing to the high controllability of degrees of freedom, ultracold atomic gases have became a versatile platform to study artificial gauge fields over the past few years [1,2]. A prominent example is spin-orbit coupling (SOC), the coupling between a particle's spin and momentum, which plays a crucial role in many fascinating phenomena such as the quantum spin Hall effect and topological superfluidity [3,4]. Theoretically, an exotic phase of matter, namely, the stripe phase, was predicted to exist in a Bose condensate with Rashba-type SOC [5,6] or Raman-laser-induced SOC [7,8]. In analogy to the long-sought supersolid phase in solid helium [9], it breaks both continuous translational symmetry to form a crystalline pattern and U(1) gauge symmetry with atoms moving frictionlessly as in a superfluid. The stripe phase is energetically favored for an intraspecies interaction strength larger than the interspecies one (e.g., $g>g_{\uparrow \downarrow}$ ), and in ${ }^{87} \mathrm{Rb}$ gas with Raman SOC, it lies in a narrow window of Rabi frequency $\Omega$ due to the small difference between $g$ and $g_{\uparrow \downarrow}[8]$. This is to be compared with the plane-wave and zero-momentum phases appearing at higher Rabi frequency. As a result, a direct observation of the stripe phase remains elusive, due to the short period and negligible density contrast of the stripes [10-13]. It has been indirectly probed using Bragg spectroscopy in a SOC Bose gas on lattices, where the interaction difference is effectively enhanced [14].

\footnotetext{
*pengshiguo@wipm.ac.cn

†phy.zoupeng@gmail.com
}

Published by the American Physical Society under the terms of the Creative Commons Attribution 4.0 International license. Further distribution of this work must maintain attribution to the author(s) and the published article's title, journal citation, and DOI.
In addition to Raman SOC, a different type of SOC, the so-called spin-orbital-angular-momentum coupling (SOAMC), was proposed recently in pioneering theoretical works [15-19]: By utilizing two copropagating LaguerreGaussian (LG) laser beams to induce an off-diagonal Stark shift, the atomic pseudospin can be coupled to its angular momentum. The resulting SOAMC is of a two-dimensional (2D) nature with an axial symmetry, which gives rise to intriguing quantum phases, such as the vortex-antivortex pair phase (see phase I in Fig. 1) with definite angular momentum $l_{z}=0$, the half-skyrmion phase II with $l_{z}=\hbar$ or $-\hbar$, and the superposition phase III with an equal-weight combination of two angular-momentum states at $l_{z}= \pm \hbar[15,16]$. These are precisely the analogs of the zero-momentum, planewave, and stripe phases in the case of Raman SOC. For this reason, phase III was previously referred to as the stripe phase $[16,17]$. The SOAMC was most recently engineered in spin-1 [20,21] and spin-1/2 ${ }^{87} \mathrm{Rb}$ Bose gases [22]. Different from the theoretical proposals [15-19], LG laser beams with a tune-out wavelength $\lambda=790.02 \mathrm{~nm}$ were used to eliminate the unnecessary diagonal Stark shift [20-22]. However, there has been no experimental observation of such a superposition phase to date. Previous theoretical studies, nevertheless, failed to address the realistic regime for this phase of most interest. By considering a quasi-two-dimensional configuration in the realistic experiment, we therefore would like to address this nontrivial phase of a SOAMC Bose gas and provide comprehensive calculations using two approaches.

In this paper we investigate ground-state phases and lowlying collective modes of a harmonically trapped pancakelike SOAMC Bose gas for realistic parameters using ${ }^{87} \mathrm{Rb}$ and ${ }^{41} \mathrm{~K}$ atoms, by developing a self-consistent Gross-Pitaevskii theory within the Bogoliubov approximation and a variational theory at zero temperature. The former theory concentrates on the case with definite angular momentum (i.e., phases I and 
II), which possesses axial symmetry, and has been intensively studied theoretically [15-19] and experimentally [20-22]. We consider the low-lying collective excitations such as the dipole and breathing modes in these quantum phases and investigate the Rabi-frequency dependence of the mode frequencies (Fig. 3). The violation of Galilean invariance of this SOAMC system is also demonstrated in the dipole mode. In the latter, we follow the idea of a variational Ansatz for the condensate wave function and probe the so-called angular stripe phase (i.e., phases III and IV) by minimizing the total mean-field energy. Quite generally, the angular stripe phase favors a double or multiple occupation of the angular-momentum states that are energetically allowed [see Fig. 2(a) and Eq. (9)] and explicitly breaks the axial symmetry of the condensate wave function. It thus possesses spatial modulation in the angular direction. This is best manifested in the angular density-density correlation function $g^{(2)}(\theta)$ for each spin state [see Figs. 6(a)-6(d)], which clearly reveals distinguishable symmetries in spatial density distributions where we have utilized the Feshbach resonance technique. We find a relatively large oscillation period and a sizable contrast in the oscillation amplitude, making direct experimental detection of the angular stripe feasible. Moreover, the appearance of these angular stripe phases can also be seen from the energetic and dynamical instabilities of the excitation spectrum.

This paper is organized as follows. We describe the model Hamiltonian and details of two theoretical methods in Sec. II. In Sec. III we present a phase diagram and density profile of distinct phases in a SOAMC gas and show the way to distinguish them. We then illustrate the Bogoliubov excitation spectrum and investigate the low-lying collective modes. Large parameter space and density modulation of the nontrivial angular stripe phases are also discussed by utilizing the Feshbach resonance technique. A summary and outlook are given in Sec. IV.

\section{THEORETICAL FRAMEWORKS}

\section{A. Model Hamiltonian}

We start by describing a setup similar to that in a recent rubidium experiment [22], where a pair of LG beams with different orbital angular momentum $\left(n_{1}=-2\right.$ and $\left.n_{2}=0\right)$ copropagate along the $z$ axis and generate the SOAMC in the $x-y$ plane. Two hyperfine states in the $F=1$ groundstate manifold of ${ }^{87} \mathrm{Rb}$ are selected to act as pseudospin states $|\uparrow\rangle$ and $|\downarrow\rangle$. For convenience, we consider a quasi-2D configuration with a highly oblate harmonic trapping potential along the $z$ direction, which allows us to tune the overall effective strength of the interatomic interactions and to enlarge the phase space for the angular stripe phase. After a unitary transformation $\mathcal{U}=\exp \left(-i n \phi \hat{\sigma}_{z}\right)$, with $n \equiv\left(n_{1}-n_{2}\right) / 2$, in the polar coordinates $(r, \phi)$ the resulting pancakelike Bose gas with SOAMC can be described by a reduced model Hamiltonian $\mathcal{H}=\mathcal{H}_{\mathrm{s}}+\mathcal{H}_{\text {int }}$, where the single-particle part reads [22]

$$
\begin{aligned}
\mathcal{H}_{\mathrm{s}}= & -\frac{\hbar^{2}}{2 m r} \frac{\partial}{\partial r}\left(r \frac{\partial}{\partial r}\right)+\frac{\left(\hat{L}_{z}-n \hbar \hat{\sigma}_{z}\right)^{2}}{2 m r^{2}} \\
& +V_{\text {ext }}(r)+\Omega_{R}(r) \hat{\sigma}_{x}-\frac{\delta}{2} \hat{\sigma}_{z}
\end{aligned}
$$

where $\hat{\sigma}_{x, z}$ are Pauli matrices, $\delta$ is the laser detuning, $V_{\text {ext }}(r)=m \omega_{r}^{2} r^{2} / 2$ is the trapping potential in the $x-y$ plane, and $\Omega_{R}(r)=\Omega(r / R)^{2} \exp \left[-2(r / R)^{2}\right]$ is the spatial-dependent coupling strength of laser beams with Rabi frequency $\Omega$ and waist $R$. The canonical angular momentum operator $\hat{L}_{z} \equiv-i \hbar \partial_{\phi}$ is thus coupled to atomic spin via the SOAMC term $\sim \hat{L}_{z} \hat{\sigma}_{z}$. Hereafter, we use the characteristic energy $\hbar \omega_{r}$, oscillator length $d=\sqrt{\hbar / m \omega_{r}}$ of the trap, and $k_{r} \equiv 1 / d$ as units of energy, length, and wave vector, respectively. We also take a relatively large waist of laser beams $R=20 d$ as in the experiments in Refs. [20,22].

In the absence of interatomic interactions, atoms occupy the single-particle states with a definite angular momentum $l_{z}$ such as $l_{z}=0$ or $\pm \hbar$, as shown in Appendix A. Taking into account interactions, at zero temperature the normalized spinor ground-state wave function $\Psi \equiv\left(\Psi_{\uparrow}, \Psi_{\downarrow}\right)$ can be determined by either self-consistently solving the Gross-Pitaevskii (GP) equation [23-25] or minimizing the mean-field energy per particle $\epsilon=\left\langle\mathcal{H}_{\mathrm{s}}+\mathcal{H}_{\text {int }}\right\rangle / N$,

$$
\begin{aligned}
\epsilon= & \iint r d r d \phi\left[\left(\Psi_{\uparrow}^{*}, \Psi_{\downarrow}^{*}\right) \mathcal{H}_{\mathrm{s}}\left(\begin{array}{c}
\Psi_{\uparrow} \\
\Psi_{\downarrow}
\end{array}\right)\right. \\
& \left.+\frac{g_{\uparrow \uparrow}}{2}\left|\Psi_{\uparrow}\right|^{4}+\frac{g_{\downarrow \downarrow}}{2}\left|\Psi_{\downarrow}\right|^{4}+g_{\uparrow \downarrow}\left|\Psi_{\uparrow}\right|^{2}\left|\Psi_{\downarrow}\right|^{2}\right] / N,
\end{aligned}
$$

which will be introduced in the following two sections, respectively.

\section{B. Gross-Pitaevskii approach within the Bogoliubov approximation}

The Gross-Pitaevskii approach is particularly useful when the condensate wave function preserves the axial symmetry and the angular momentum $l_{z}$ remains as a good quantum number, reading

$$
\psi_{l_{z}}(r, \phi)=\left(\begin{array}{c}
\varphi_{l_{z} \uparrow}(r) \\
\varphi_{l_{z} \downarrow}(r)
\end{array}\right) \frac{e^{i l_{z} \phi}}{\sqrt{2 \pi}} .
$$

In this case, we generalize the Bogoliubov theory at zero temperature to study low-energy elementary excitations [26,27], including dipole and breathing modes. The transitions between different phases may then be understood from possible instabilities of the collective modes.

In a grand canonical ensemble, the Heisenberg equations of motion for the Bose operator field $\hat{\Psi}(r, \phi, t)=\left(\hat{\Psi}_{\uparrow}, \hat{\Psi}_{\downarrow}\right)^{T}$ take the form

$$
i \hbar \frac{\partial}{\partial t} \hat{\Psi}=\left[\mathcal{H}_{\mathrm{s}}-\mu+\operatorname{diag}\left(\hat{\mathcal{L}}_{\uparrow}, \hat{\mathcal{L}}_{\downarrow}\right)\right] \hat{\Psi},
$$

with the operator $\hat{\mathcal{L}}_{\sigma} \equiv g_{\sigma \sigma} \hat{\Psi}_{\sigma}^{\dagger} \hat{\Psi}_{\sigma}+g_{\uparrow \downarrow} \hat{\Psi}_{\bar{\sigma}}^{\dagger} \hat{\Psi}_{\bar{\sigma}}$ and the chemical potential $\mu$. Following the standard procedure [23,24,28], the Bose field operator $\hat{\Psi}_{\sigma}(r, \phi, t)$ for spin component $\sigma=$ $\uparrow, \downarrow$ can be rewritten as a combination of the condensate wave function $\psi_{l_{z} \sigma}$ and the noncondensate fluctuation operator $\hat{\eta}_{\sigma}$ with

$$
\begin{aligned}
\hat{\Psi}_{\sigma}(r, \phi, t) & =\psi_{l_{z} \sigma}(r, \phi)+\hat{\eta}_{\sigma}(r, \phi, t), \\
\hat{\eta}_{\sigma}(r, \phi, t) & =\sum_{j}\left[u_{j \sigma} e^{-i \varepsilon_{j} t} \hat{\alpha}_{j}+v_{j \sigma}^{*} e^{i \varepsilon_{j} t} \hat{\alpha}_{j}^{\dagger}\right],
\end{aligned}
$$


where we assume the static condensate wave function $\psi_{l_{z} \sigma}(r, \phi)$ with a definite angular momentum $l_{z}$ as in Eq. (3) and the fluctuation operator $\hat{\eta}_{\sigma}$ is expanded in a quasiparticle basis $\left(\hat{\alpha}^{\dagger}, \hat{\alpha}\right)$ under a Bogoliubov transformation. In addition, $\varepsilon_{j}$ is the quasiparticle frequency and the quasiparticle amplitudes $\mathcal{K} \equiv u$ (or $v$ ) in Eq. (5) can be written as $\mathcal{K}_{j \sigma}(r, \phi)=$ $\mathcal{K}_{\tau \sigma}^{(l)}(r) r^{\left|l_{z}+l\right|} e^{i\left(l_{z}+l\right) \phi} / \sqrt{2 \pi}$ [27]. Here the index $j$ is defined as $j \equiv(l, \tau)$, with the integer angular momentum $l \in \mathbb{Z}$ and the branch index $\tau \in \mathbb{N}_{1}$.

After substituting the new Bose field operator (5) into the equations of motion (4) and applying the mean-field decoupling for the three-operator terms [24], we obtain two coupled equations. The first is the modified GP equation for the static condensate

$$
\left[\mathcal{H}_{\mathrm{s}}\left(\hat{L}_{z}\right)+\operatorname{diag}\left(\mathcal{L}_{\uparrow}, \mathcal{L}_{\downarrow}\right)\right] \psi_{l_{z}}=\mu \psi_{l_{z}},
$$

with the spinor $\psi_{l_{z}} \equiv\left(\psi_{l_{z} \uparrow}, \psi_{l_{z} \downarrow}\right)^{T}$ and the diagonal element $\mathcal{L}_{\sigma} \equiv g_{\sigma \sigma} n_{\sigma}+g_{\uparrow \downarrow} n_{\bar{\sigma}}$ (here the spin index $\sigma \neq \bar{\sigma}$ ). The other one is the coupled Bogoliubov equation for quasiparticles

$$
\begin{aligned}
{\left[\mathcal{H}_{\mathrm{s}}\left(\hat{L}_{z}\right)+\mathcal{A}_{\uparrow}\right] U_{j}+\mathcal{B} V_{j} } & =\varepsilon_{j} U_{j}, \\
-\mathcal{B} U_{j}^{*}-\left[\mathcal{H}_{\mathrm{s}}\left(\hat{L}_{z}\right)+\mathcal{A}_{\downarrow}^{*}\right] V_{j}^{*} & =\varepsilon_{j} V_{j}^{*},
\end{aligned}
$$

where $U_{j} \equiv\left(u_{j \uparrow}, u_{j \downarrow}\right)^{T}, V_{j} \equiv\left(v_{j \uparrow}, v_{j \downarrow}\right)^{T}$, and

$$
\begin{aligned}
\mathcal{A}_{\sigma} & \equiv\left[\begin{array}{cc}
2 g_{\uparrow \uparrow} n_{\uparrow}+g_{\uparrow \downarrow} n_{\downarrow} & g_{\uparrow \downarrow} \psi_{l_{z} \sigma} \psi_{l_{z} \bar{\sigma}}^{*} \\
g_{\uparrow \downarrow} \psi_{l_{z} \bar{\sigma}} \psi_{l_{z} \sigma}^{*} & 2 g_{\downarrow \downarrow} n_{\downarrow}+g_{\uparrow \downarrow} n_{\uparrow}
\end{array}\right], \\
\mathcal{B} & \equiv\left[\begin{array}{cc}
g_{\uparrow \uparrow} \psi_{l_{z} \uparrow}^{2} & g_{\uparrow \downarrow} \psi_{l_{z} \uparrow} \psi_{l_{z} \downarrow} \\
g_{\uparrow \downarrow} \psi_{l_{z} \uparrow} \psi_{l_{z} \downarrow} & g_{\downarrow \downarrow} \\
l_{l_{z} \downarrow}^{2}
\end{array}\right] .
\end{aligned}
$$

Here $n_{\sigma}=\left|\psi_{l_{z} \sigma}\right|^{2}$ denotes the density for spin component $\sigma$ with $\sigma \neq \bar{\sigma}$. Note that, in the GP and Bogoliubov equations, the noncondensate density is neglected at zero temperature since the quantum depletion is typically negligible.

Therefore, the wave function or relevant energy of the states with different angular momentum can be obtained by self-consistently solving the GP equation. After numerically calculating the ground-state wave function $\psi_{l_{z}}(r, \phi)$, one obtains straightforwardly the elementary excitations $\varepsilon_{j}$ with respect to the quasiparticle angular momentum $l$ via the Bogoliubov equations (7).

\section{Variational approach}

When the axial symmetry is spontaneously broken by interactions (for instance, in the cases of stripe phases), the angular momentum $l_{z}$ is no longer a good quantum number. Thus, we instead consider a variational Ansatz for the condensate wave function and determine the ground state within the energy-minimizing approach using Eq. (2). In this case [i.e., $\left.n=\left(n_{1}-n_{2}\right) / 2=-1\right]$, we adopt the variational Ansatz

$$
\boldsymbol{\Psi}(r, \phi)=\alpha e^{i \theta_{\alpha}} \psi_{-1}+\beta e^{i \theta_{\beta}} \psi_{0}+\gamma e^{i \theta_{\gamma}} \psi_{1},
$$

where $\psi_{l_{z}}$ is the definite-angular-momentum state in Eq. (3) solved from the self-consistent GP equation with the interaction effect incorporated, as described in Sec. II B. In addition, $\alpha, \beta$, and $\gamma$ are real non-negative weighting coefficients and $\theta_{\alpha}, \theta_{\beta}$, and $\theta_{\gamma}$ are the phases. Thus, the mean-field energy $\epsilon$ becomes a functional of three variational parameters if we take into consideration the normalization condition $\alpha^{2}+$ $\beta^{2}+\gamma^{2}=1$ and the fact that $\epsilon$ depends on the phase factors through a single function $\cos \left(\theta_{\alpha}-2 \theta_{\beta}+\theta_{\gamma}\right)$ only (see also Appendix A). The wave function $\boldsymbol{\Psi}$ is then determined from the minimization of $\epsilon$. We have checked that the use of more definite-angular-momentum states in the variational Ansatz does not result in appreciable improvement for lowering $\epsilon$. It is worth noting that the above Ansatz in terms of the definite-angular-momentum states possesses a lower energy than the one in terms of the single-particle states, which is rather significant at relatively large interaction strength $g$ in the low-Rabi-frequency limit, as shown in Appendix A.

\section{RESULTS AND DISCUSSION}

\section{A. Phase diagram}

In experiments with ${ }^{87} \mathrm{Rb}$ and ${ }^{23} \mathrm{Na}$ atoms in pancake traps [29-31], the interaction strengths $g_{\uparrow \uparrow}=g_{\downarrow \downarrow}=g \equiv$ $\sqrt{8 \pi}\left(a / a_{z}\right) \hbar^{2} / m$ and $g_{\uparrow \downarrow} \equiv \sqrt{8 \pi}\left(a_{\uparrow \downarrow} / a_{z}\right) \hbar^{2} / m$ span approximately over the range $[0.01,0.15] \hbar^{2} / \mathrm{m}$ and cross from the weakly interacting to relatively strongly interacting regimes. Here $a$ and $a_{\uparrow \downarrow}$ are, respectively, the intra- and interspecies $s$-wave scattering lengths in three dimensions and $a_{z}=$ $\sqrt{\hbar / m \omega_{z}}$ is the oscillator length along the tightly confined $z$ axis.

In Fig. 1 we report the general phase diagram at a given intraspecies interaction strength $g$ [Fig. 1(a)] or at a fixed ratio $g_{\uparrow \downarrow} / g$ [Fig. 1(b)]. At an experimentally accessible interaction strength $m g / \hbar^{2} \sim 0.01$ in Fig. 1(a) [31], by decreasing $g_{\uparrow \downarrow} / g$ from 1 we find that the angular stripe phase III becomes favorable very soon, similar to the stripe phase in a Raman SOC Bose gas. In contrast, the angular stripe phase IV is less sensitive to the ratio and its parameter space is nearly unchanged upon deceasing $g_{\uparrow \downarrow} / g$. At a typical ratio $g_{\uparrow \downarrow} / g=0.5$ shown in Fig. 1(b), we examine the dependence of different phases on the overall interaction strength. With Raman SOC, it is known that a large interaction strength enhances the stripe and zero-momentum phases and suppresses the planewave phase, leading to a tricritical point where three phases

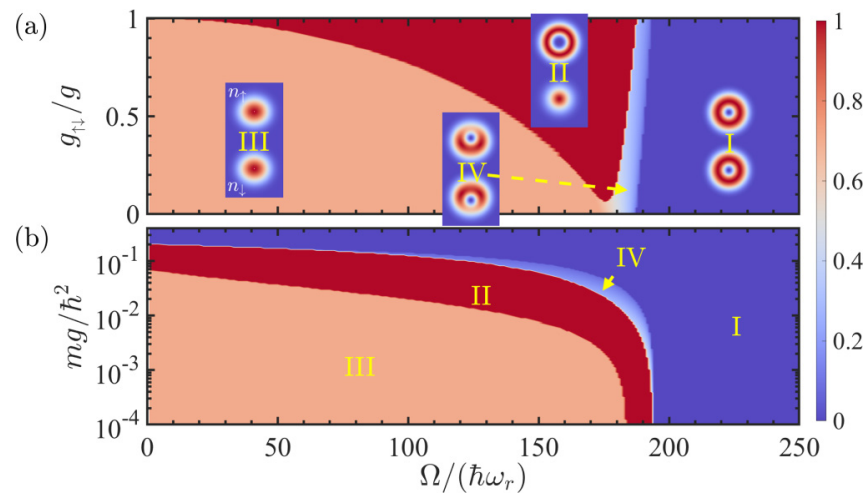

FIG. 1. Phase diagram of a harmonically trapped pancakelike SOAMC Bose gas with particle number $N=10^{3}$ (a) in the $g_{\uparrow \downarrow} / g-\Omega$ plane at $m g / \hbar^{2}=0.01$ and (b) in the $g-\Omega$ plane at $g_{\uparrow \downarrow} / g=0.5$. The color indicates the portion of the $l_{z}=\hbar$ state in the condensate wave function. The insets show typical densities for each spin state in different phases. The phase boundaries are discussed in the text. 


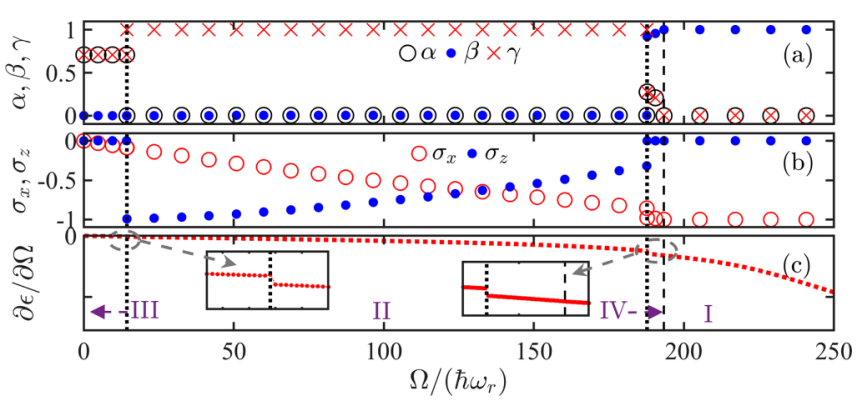

FIG. 2. (a) Weighting coefficients $\alpha, \beta$, and $\gamma$, (b) spin polarizations $\left\langle\sigma_{x}\right\rangle$ and $\left\langle\sigma_{z}\right\rangle$, and (c) the derivative of the mean-field energy $\partial \epsilon / \partial \Omega$, as a function of $\Omega$ at $g=0.01 \hbar^{2} / m$, for a pancake SOAMC ${ }^{87} \mathrm{Rb}$ Bose gas with $g_{\uparrow \downarrow} / g=100.40 / 100.86$ and $N=10^{3}$. The vertical black dotted (dashed) lines indicate the first-order (second-order) phase transitions.

intervene [8]. Here we find that only the vortex-antivortex pair phase I survives at large interaction strength. The large parameter space of the phase I can be understood from the absence of the diagonal Stark shift in the recent experiments, which removes an additional confinement $\Omega_{R}(r)$ to both spin components [20,22] and hence makes the zero-angularmomentum state $\psi_{0}$ more energetically favorable by reducing the interaction energy.

In Fig. 2 we present the variational results for a Bose gas of $N=10^{3}{ }^{87} \mathrm{Rb}$ atoms with $g_{\uparrow \downarrow} / g=a_{\uparrow \downarrow} / a=$ $100.40 / 100.86$ [22] at a typical interaction strength $m g / \hbar^{2}=$ 0.01 . By decreasing the Rabi frequency $\Omega$ from high values, we may identify four distinct regimes: (i) the vortex-antivortex pair phase I with zero angular momentum $l_{z}=0$ (i.e., $\beta=1$ and $\alpha=\gamma=0$ ), $\left\langle\sigma_{z}\right\rangle=0$, and $\left\langle\sigma_{x}\right\rangle=-1$; (ii) the angular stripe phase IV with no definite $l_{z}$ (i.e., $\beta \neq 0$ and $\alpha=\gamma \neq$ 0 ), $\left\langle\sigma_{z}\right\rangle=0$, and $\left\langle\sigma_{x}\right\rangle \neq 0$; (iii) the half-skyrmion phase II with $l_{z}=-\hbar$ or $\hbar$ (i.e., $\alpha=1$ and $\beta=\gamma=0$, or $\gamma=1$ and $\alpha=\beta=0$ ), $\left\langle\sigma_{z}\right\rangle \neq 0$, and $\left\langle\sigma_{x}\right\rangle \neq 0$; and (iv) the angular stripe phase III, which may be viewed as a specific case of the phase IV but with $\beta=0$ and $\alpha=\gamma=1 / \sqrt{2}$. Compared with the results of Raman-induced or Rashba-type SOC [5,8], it is readily seen that phases III, II, and I are in a one-to-one correspondence with the well-known stripe, plane-wave, and zero-momentum phases, respectively $[16,17]$. The angular stripe phase IV is unique to a SOAMC Bose gas, as a result of the discreteness of the angular momentum.

The nature of the transition between different superfluid phases may be characterized by calculating the derivative of the mean-field energy with respect to the Rabi frequency [16,17], $\partial \epsilon / \partial \Omega$, as shown in Fig. 2(c) with vertical dashed and dotted lines for the second- and first-order transitions, respectively. We find a first-order transition from the angular stripe phase III to the half-skyrmion phase II, the same as the transition between the stripe and plane-wave phases in a Raman SOC Bose gas [8,32]. The transition from phase II to the angular stripe phase IV is also of first order, accompanied by the sudden appearance of the component $\psi_{0}$ in the condensate wave function $\boldsymbol{\Psi}$ (i.e., $\beta \neq 0$ ) and sudden change in the spin polarizations. In contrast, the transition from the angular stripe phase IV to the vortex-antivortex pair phase I is continuous and the weighting coefficients $\alpha$ and $\gamma$ disappear gradually close to the transition.

\section{B. Collective modes and instabilities}

We now turn to discuss the low-lying collective excitations, which are readily measurable in SOC Bose gases [33-35]. In Figs. 3(a) and 3(b) typical excitation spectra in the halfskyrmion phase II and the vortex-antivortex pair phase I are plotted as a function of the angular momentum $l$ of Bogoliubov quasiparticles, respectively. The Goldstone mode (i.e., condensate mode), low- and high-dipole modes (with $l= \pm \hbar$ ), and breathing mode (i.e., the lowest $l=0$ mode) are indicated by crosses, asterisks, circles, and inverted triangles, respectively. In phase II we observe a clear roton structure, despite the discreteness of the spectrum, similar to the roton found in the plane-wave phase of a Raman SOC Bose gas [28,32,34-36]. This originates from the spontaneous breaking of the axial symmetry and explains the first-order III-II phase transition we mentioned earlier.

In Fig. 3(c), a few low-lying mode frequencies are shown as a function of $\Omega$. The $\Omega$ dependence of the low-dipole mode frequency (asterisks) is of particular interest and exhibits an intriguing behavior. By decreasing the Rabi frequency from the vortex-antivortex pair phase I, we find that the low-dipole mode frequency becomes vanishingly low at $\Omega \simeq 197.5 \hbar \omega_{r}$, which is close to the I-IV phase boundary $\Omega_{c} \simeq 193.3 \hbar \omega_{r}$ determined from the variational calculations. As $\Omega$ decreases further, the mode frequency shows a jump, then increases steadily, and finally saturates at about $0.62 \omega_{r}$ at $\Omega=0$. This interesting $\Omega$ dependence of the dipole mode was observed earlier in a SOC Bose gas, where the complete softening of the mode occurs at the transition from the zero-momentum to plane-wave phases and is associated with the divergent effective mass or magnetic susceptibility at the transition $[33,37,38]$. In our case, although the mode frequency becomes discrete due to the quantization of angular momentum, the feature of complete softening remains. It is also a clear demonstration of the violation of Galilean
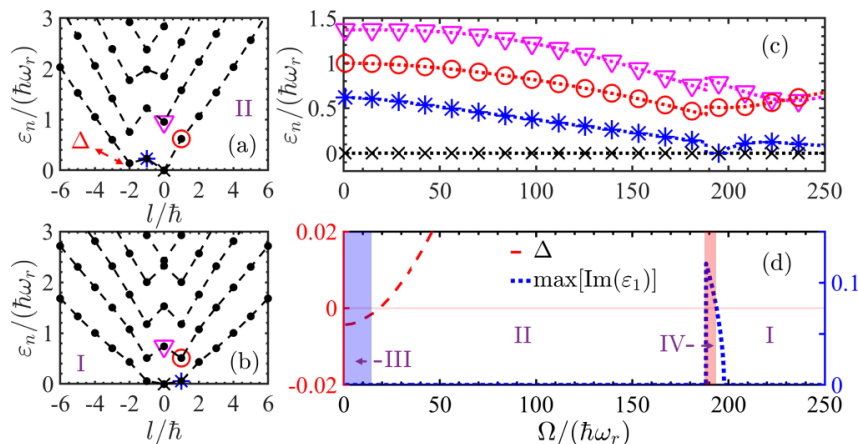

FIG. 3. Excitation spectrum as a function of the angular momentum $l$ of Bogoliubov quasiparticles at (a) $\Omega / \hbar \omega_{r}=150$ and (b) $\Omega / \hbar \omega_{r}=200$. The roton minimum is denoted by $\Delta$. (c) and (d) Mode frequencies $\varepsilon_{n}(l)$, roton energy $\Delta$, and maximum imaginary part of the lowest branch $\varepsilon_{n=1}(l)$ as a function of Rabi frequency $\Omega$. The blue and red shaded areas highlight the regimes of the angular stripe phases III and IV, respectively, as determined from Fig. 2. The other parameters are the same as in Fig. 2. 
invariance due to SOAMC [36]. On the other hand, the frequencies of the breathing mode (inverted triangles) and the high-dipole mode (circles) both experience a jump at the transition from the angular stripe phase IV to the phase II and sequentially increase upon decreasing $\Omega$. Near zero Rabi frequency, the breathing mode frequency is about $1.37 \omega_{r}$, lower than the scale-invariant classical prediction of $2 \omega_{r}$ [39] due to relatively large interatomic interactions. The high-dipole mode frequency approaches $\omega_{r}$, since the SOAMC can be gauged away in the $\Omega \rightarrow 0$ limit and the Galilean invariance can be restored, ensuring the exact solution of the Kohn mode with frequency $\omega_{r}$. At sufficiently high Rabi frequency, the mode frequencies in the same branch tend to approach each other since the low-lying excitation bands get flattened [16].

We note that, close to the I-IV transition, a non-negligible imaginary part appears in the lowest excitation branch, as shown by the blue dotted curve in Fig. 3(d). This is simply an indication of the dynamical instability of the vortex-antivortex pair phase towards the phase transition. Furthermore, close to the III-II transition, the roton gap $\Delta$ [red dashed curve in Fig. 3(d)] starts to become negative, indicating the energetic instability of the phase II and thus determining a low bound for the III-II transition [13]. Two unstable regimes seen from the collective modes agree qualitatively with the red and blue shaded areas of the angular stripe phases IV and III determined using the variational approach.

\section{Experimental detection of the angular stripe phase}

The atomic interaction is one of the crucial factors to affect the parameter space of the angular stripes as well as their visibility in current atomic experiments. To this end, let us consider a Feshbach resonance (FR) centered at the magnetic field $B_{0}=51.95 \mathrm{G}$ of ${ }^{41} \mathrm{~K}$ atomic gases. Near the FR, the intraspecies scattering lengths $\left(a_{\uparrow \uparrow}, a_{\downarrow \downarrow}\right) \simeq(65,100) a_{0}$ are approximately constant as shown in Fig. 4, where $a_{0}$ is the Bohr radius, and the interspecies one $a_{\uparrow \downarrow}$ can be tuned in a wide range [40,41]. In a realistic quasi-2D harmonic trap with frequencies $\left(\omega_{r}, \omega_{z}\right) / 2 \pi=(10,200) \mathrm{Hz}$ and an aspect ratio $\omega_{z} / \omega_{r}=20$, the effective 2D interaction strengths become $m g_{\uparrow \uparrow} / \hbar^{2}=0.015$ and $m g_{\downarrow \downarrow} / \hbar^{2}=0.023$, respectively. For the sake of illustration, hereafter we will only present the calcu-

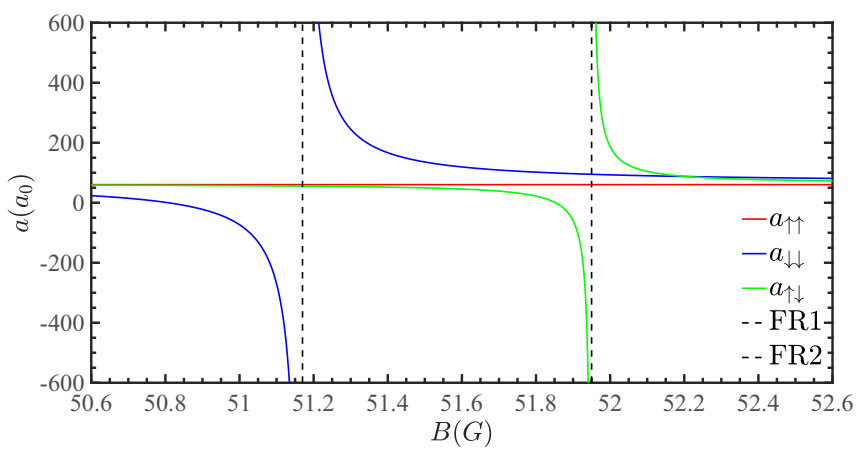

FIG. 4. Feshbach resonance of ${ }^{41} \mathrm{~K}$ atoms at a magnetic field about $B_{0}=51.95 \mathrm{G}$, in the vicinity of which the intraspecies scattering lengths $a_{\uparrow \uparrow}$ and $a_{\downarrow \downarrow}$ are almost constant while the interspecies one $a_{\uparrow \downarrow}$ is tunable [40,41].

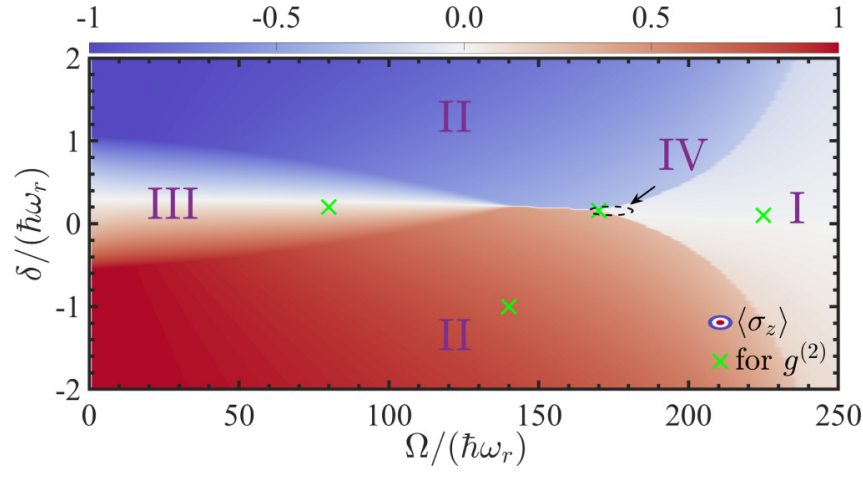

FIG. 5. Phase diagram in the $\delta-\Omega$ plane of a SOAMC ${ }^{41} \mathrm{~K}$ gas, in harmonic traps $\left(\omega_{r}, \omega_{z}\right)=2 \pi \times(10,200) \mathrm{Hz}$ with $N=10^{3}$ and $\left(a_{\uparrow \uparrow}, a_{\downarrow \downarrow}, a_{\uparrow \downarrow}\right)=(65,100,10) a_{0}$. The color indicates the expectation value of spin magnetization $\left\langle\sigma_{z}\right\rangle$ in the condensate wave function. The green crosses indicate the selected positions for four distinct phases in Fig. 6, respectively.

lations at a typical interspecies scattering length $a_{\uparrow \downarrow}=10 a_{0}$ (i.e., tuned by a magnetic field $B=51.83 \mathrm{G}$ in the laboratory). In Fig. 5 we show the phase diagram in the $\delta-\Omega$ plane by visualizing the spin magnetization $\left\langle\sigma_{z}\right\rangle$ which is frequently measured in experiments. In general, the spin magnetization exhibits distinct behaviors in different phases. Phase I lies in the gray region at relatively high $\Omega$ with $\left\langle\sigma_{z}\right\rangle \sim 0$. Phase II is indicated by the red and blue colors representing the definiteangular-momentum states $\psi_{-1}$ and $\psi_{1}$ with $\left\langle\sigma_{z}\right\rangle$ smoothly varying from 1 and from -1 , respectively. Stripe phase III (i.e., $\left.\psi_{-1}+\psi_{1}\right)$ lies in the gradient color region at relatively low $\Omega$ with $\left\langle\sigma_{z}\right\rangle$ changing significantly from 1 to -1 . Stripe phase IV (i.e., $\psi_{-1}+\psi_{0}+\psi_{1}$ ) lies in a tiny gray region at high $\Omega$ denoted by a dashed circle. It is readily seen that stripe phase III occupies a relatively large window of $1.5 \hbar \omega_{r}$ by tuning the detuning $\delta$, which corresponds to a range of about $100 \mathrm{~Hz}$ in frequency and hence can be easily operated in experiments. This large parameter space of stripe phases can also be seen in Appendix B, where the supplementary calculations of the weights of various $l_{z}$ states at different realistic values of $a_{\uparrow \downarrow}$ are discussed.

To estimate the visibility in the density profile, we introduce an angular density-density correlation function

$$
g_{i}^{(2)}(\theta) \equiv \frac{\int_{0}^{2 \pi} n_{i}(\phi) n_{i}(\phi+\theta) d \phi}{\int_{0}^{2 \pi} n_{i}^{2}(\phi) d \phi},
$$

with the angular density $n_{i}(\phi)=\int_{0}^{\infty} r d r n_{i}(r, \phi)$ and the label $i=\uparrow, \downarrow$ for each spin component and null for the total density. As shown in Figs. 6(a) and 6(b), the correlation $g^{(2)}(\theta)$ in the vortex-antivortex pair phase I and the half-skyrmion phase II is identically unity due to the axial symmetry of the phases, being two vortices and one vortex plus one ThomasFermi distribution, respectively. In contrast, the angular stripe phases break the axial symmetry and exhibit spatial modulation in the directional angle $\theta$. In stripe phase IV [Fig. 6(d)], the modulation $1-g^{(2)}(\theta)$ is relatively small (i.e., about 0.08 ) due to the large portion of the $\psi_{0}$ state in the condensate wave function [see also Fig. 2(a)]. In sharp contrast, angular stripe phase III shows a much larger spatial oscillation in $1-g^{(2)}(\theta)$ 

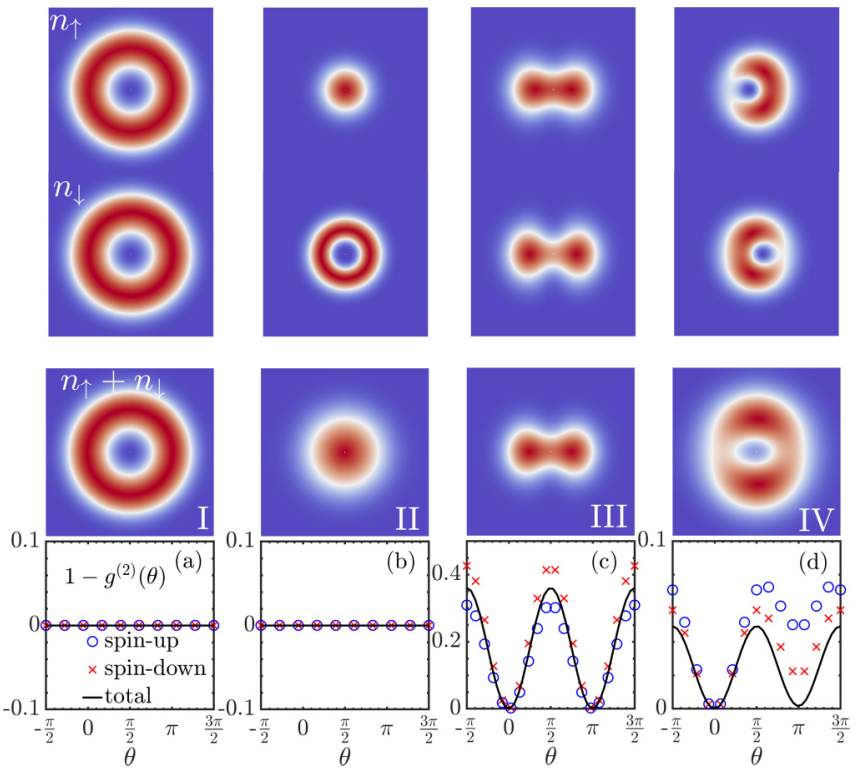

FIG. 6. Shown in the top three panels are typical spin-component total density profiles in phases I-IV from left to right. (a)-(d) The bottom panel shows the corresponding angular density-density correlation function $1-g^{(2)}(\theta)$ for different phases. The results for spinup, spin-down, and total density are indicated by circles, crosses, and solid lines, respectively. These four examples are taken at the green crosses in Fig. 5.

up to about 0.4 for both spin components, as can be seen in Fig. 6(c). This hallmark feature might be useful in directly probing the existence of the angular stripe in experiments.

\section{CONCLUSION AND OUTLOOK}

In summary, we have predicted the existence of two angular stripe phases in a Bose condensate of ${ }^{87} \mathrm{Rb}$ with spinorbital-angular-momentum coupling, which feature occupation of different angular-momentum states and have sizable spatial modulation in the angular density-density correlation. The phase space for these superfluids is notable and insensitive to the difference in the intra- and interspecies interactions. A Bose gas of ${ }^{41} \mathrm{~K}$ atoms in pancake traps could be a promising candidate system to probe the predicted stripe phase.

\section{ACKNOWLEDGMENTS}

We acknowledge fruitful discussions with Yun Li and Ivana Vasić. Our research was supported by the National Natural Science Foundation of China through Grants No. 11974384 (S.-G.P.) and No. 11804177 (P.Z.), the National Key Research and Development Program Grant No. 2016YFA0301503 (S.-G.P.), the Shandong Provincial Natural Science Foundation (China) Grant No. ZR2018BA032 (P.Z.), and the Australian Research Councils Discovery Projects No. FT140100003 and No. DP180102018 (X.-J.L.) and Projects No. FT130100815 and No. DP170104008 (H.H.).
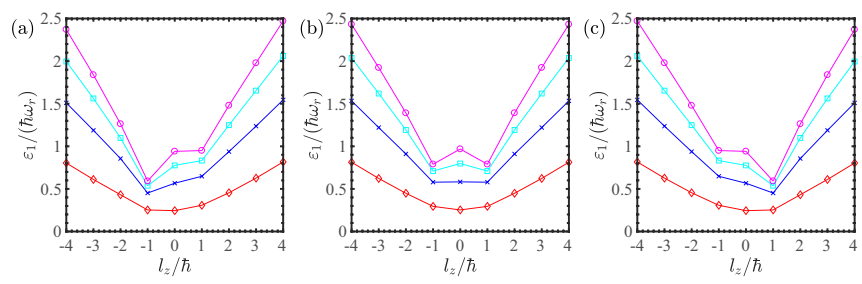

FIG. 7. Lowest branch $\varepsilon_{n=1}$ in the single-particle dispersion at (a) negative, (b) zero, and (c) positive laser detuning $\delta$ as a function of the angular momentum $l_{z}$ when the Rabi frequency $\Omega$ increases (top to bottom).

\section{APPENDIX A: SINGLE-PARTICLE DISPERSION AND COMPARISON BETWEEN TWO DIFFERENT VARIATIONAL ANSÄTZE}

We start with the single-particle Hamiltonian $\mathcal{H}_{\mathrm{s}}$ given in Eq. (1) in the $r$ - $\phi$ plane. The axial symmetry makes the angular momentum $l_{z}$ a good quantum number, and we can calculate the single-particle states $\psi_{l_{2}}^{(0)}$ as well as the dispersion relation $\varepsilon_{n}$ by employing the Schrödinger equation

$$
\mathcal{H}_{\mathrm{s}} \psi_{l_{z}}^{(0)}(r, \phi)=\varepsilon_{n} \psi_{l_{z}}^{(0)}(r, \phi) \text {. }
$$

In Fig. 7 we depict the lowest energy level $\varepsilon_{n=1}\left(l_{z}\right)$ as a function of $l_{z}$. At zero detuning $\delta=0$ [see Fig. 7(b)], as the Rabi frequency $\Omega$ decreases (from bottom to top), the dispersion changes from a structure with a single minimum at $l_{z}=0$ to that with two degenerate minima at $l_{z}= \pm \hbar$. Once the detuning of the lasers is nonzero, the energy dispersion becomes asymmetric. As we decrease $\Omega$, the angular momentum of the condensate at the minimum changes from $l_{z}=0$ to $l_{z}=\hbar(-\hbar)$ for $\delta>0(\delta<0)$.

In the presence of interactions, the total Hamiltonian reads $\mathcal{H}=\mathcal{H}_{\mathrm{s}}+\mathcal{H}_{\text {int }}$. We may assume that the angular momentum $l_{z}$ is still a good quantum number and determine the definiteangular-momentum (DAM) states $\psi_{l_{z}}^{\text {(DAM) }}$ by self-consistently solving the nonlinear Schrödinger equation or the GrossPitaevskii equation [see also Eq. (6)]

$$
\mathcal{H} \psi_{l_{z}}^{(\mathrm{DAM})}(r, \phi)=\varepsilon_{n} \psi_{l_{z}}^{(\mathrm{DAM})}(r, \phi) .
$$

In the variational approach, we can start with a variational Ansatz for the condensate wave function, i.e., a linear superposition of the lowest angular-momentum states $\psi_{l_{z}}$, which can be either the single-particle states $\psi_{l_{z}}^{(0)}$ or the self-consistently calculated definite-angular-momentum states $\psi_{l_{z}}^{(\mathrm{DAM})}$. The latter choice of course is more favorable, as the interaction effect is already included at the beginning. Explicitly, the condensate wave function is given by

$$
\boldsymbol{\Psi}(r, \phi)=\alpha e^{i \theta_{\alpha}} \psi_{-1}+\beta e^{i \theta_{\beta}} \psi_{0}+\gamma e^{i \theta_{\gamma}} \psi_{1}
$$

in terms of three phase angles $\theta_{\alpha}, \theta_{\gamma}$, and $\theta_{\beta}$ and three real non-negative parameters $\alpha, \beta$, and $\gamma$ satisfying the normalization condition $\alpha^{2}+\beta^{2}+\gamma^{2}=1$. The mean-field energy functional in terms of $\Psi(r, \phi) \equiv\left(\Psi_{\uparrow}, \Psi_{\downarrow}\right)^{T}$ is then written as

$$
E_{\mathrm{MF}}=\iint r d r d \phi\left[\boldsymbol{\Psi}^{\dagger} \mathcal{H}_{\mathrm{s}}\left(\hat{L}_{z}\right) \boldsymbol{\Psi}+\sum_{\sigma, \sigma^{\prime}} \frac{g_{\sigma \sigma^{\prime}}}{2} \Psi_{\sigma}^{\dagger} \Psi_{\sigma^{\prime}}^{\dagger} \Psi_{\sigma^{\prime}} \Psi_{\sigma}\right] .
$$



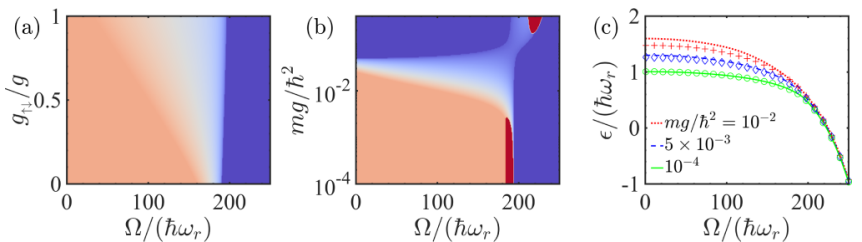

FIG. 8. Phase diagram in (a) the $g_{\uparrow \downarrow} / g-\Omega$ plane and (b) the $g-\Omega$ plane, obtained by using the variational Ansatz, which is constructed by using the single-particle states $\psi_{l_{z}}^{(0)}$. The parameters are the same as in Fig. 1. The color indicates the portion of the $l_{z}=\hbar$ state in the condensate wave function. (c) Mean-field energy $\epsilon \equiv E_{\mathrm{MF}} / N$ as a function of $\Omega$, at three sets of interaction strength $g$, calculated by using single-particle states (lines) and self-consistently determined states (symbols) in the variational Ansatz.

In particular, the interaction energy per particle $\epsilon_{\text {int }}$ reads

$$
\epsilon_{\text {int }}=\frac{1}{2 N} \iint r d r d \phi\left[g_{\uparrow \uparrow} n_{\uparrow} n_{\uparrow}+g_{\downarrow \downarrow} n_{\downarrow} n_{\downarrow}+2 g_{\uparrow \downarrow} n_{\uparrow} n_{\downarrow}\right],
$$

where we have introduced the density $n_{\sigma}(r, \phi) \equiv\left|\Psi_{\sigma}(r, \phi)\right|^{2}$ for spin component $\sigma=\uparrow, \downarrow$. The integral of the density terms $n_{\sigma} n_{\sigma^{\prime}}$ depends on the phase angles $\theta_{\alpha}, \theta_{\beta}$, and $\theta_{\gamma}$ through a cosine function $\cos \left(\theta_{\alpha}-2 \theta_{\beta}+\theta_{\gamma}\right)$. Therefore, the new angle $\Theta \equiv \theta_{\alpha}-2 \theta_{\beta}+\theta_{\gamma}$ can take an arbitrary value in the range $[0,2 \pi]$ if the coefficient in front of the cosine function vanishes. Otherwise, $\Theta$ can only take values of 0 or $\pi$ depending on the sign of the nonzero coefficient. Hence, the variational wave function of the condensed phase and relevant observables such as the spin polarizations can be obtained straightforwardly after the minimization of the energy functional with respect to these variational parameters.

In Figs. 8(a) and 8(b) we show, respectively, the phase diagram in the $g_{\uparrow \downarrow} / g-\Omega$ and $g$ - $\Omega$ planes obtained by using the variational Ansatz constructed from the single-particle states $\psi_{l_{z}}^{(0)}$, which are to be compared with the ones constructed from the definite-angular-momentum states $\psi_{l_{2}}^{\text {(DAM) }}$ as shown in Fig. 1. The two results are in quantitative agreement at sufficiently small interaction strength (i.e., $g \lesssim 10^{-3} \hbar^{2} / \mathrm{m}$ ). However, as the interactions become large, there are significant differences in the phase diagrams. These differences can be understood by comparing their mean-field energy $\epsilon$ as shown in Fig. 8(c). The two energies agree very well at small $g$. Nonetheless, they start to become different as $g$ continues to increase. The difference is much more pronounced at low Rabi frequency: The energy calculated using $\psi_{l_{z}}^{\text {(DAM) }}$ (symbols) appears to be lower than the one calculated using $\psi_{l_{2}}^{(0)}$ (lines). Therefore, for the results discussed in the main text we adopt the variational Ansatz constructed in terms of $\psi_{l_{z}}^{\text {(DAM) }}$.

\section{APPENDIX B: MORE EXAMPLES OF $a_{\uparrow \downarrow}$ NEAR THE ${ }^{41} \mathrm{~K}$ FESHBACH RESONANCE}

In Sec. IIIC a Feshbach resonance of a harmonically trapped SOAMC ${ }^{41} \mathrm{~K}$ gas at a magnetic field $B_{0}=51.95$ $\mathrm{G}$ was utilized where $a_{\uparrow \uparrow}=65 a_{0}$ and $a_{\downarrow \downarrow}=100 a_{0}$ are approximately constant near the FR and $a_{\uparrow \downarrow}$ can be tuned by FR [40,41] (see also Fig. 4). In the main text we considered a variational Ansatz for the ground-state wave function
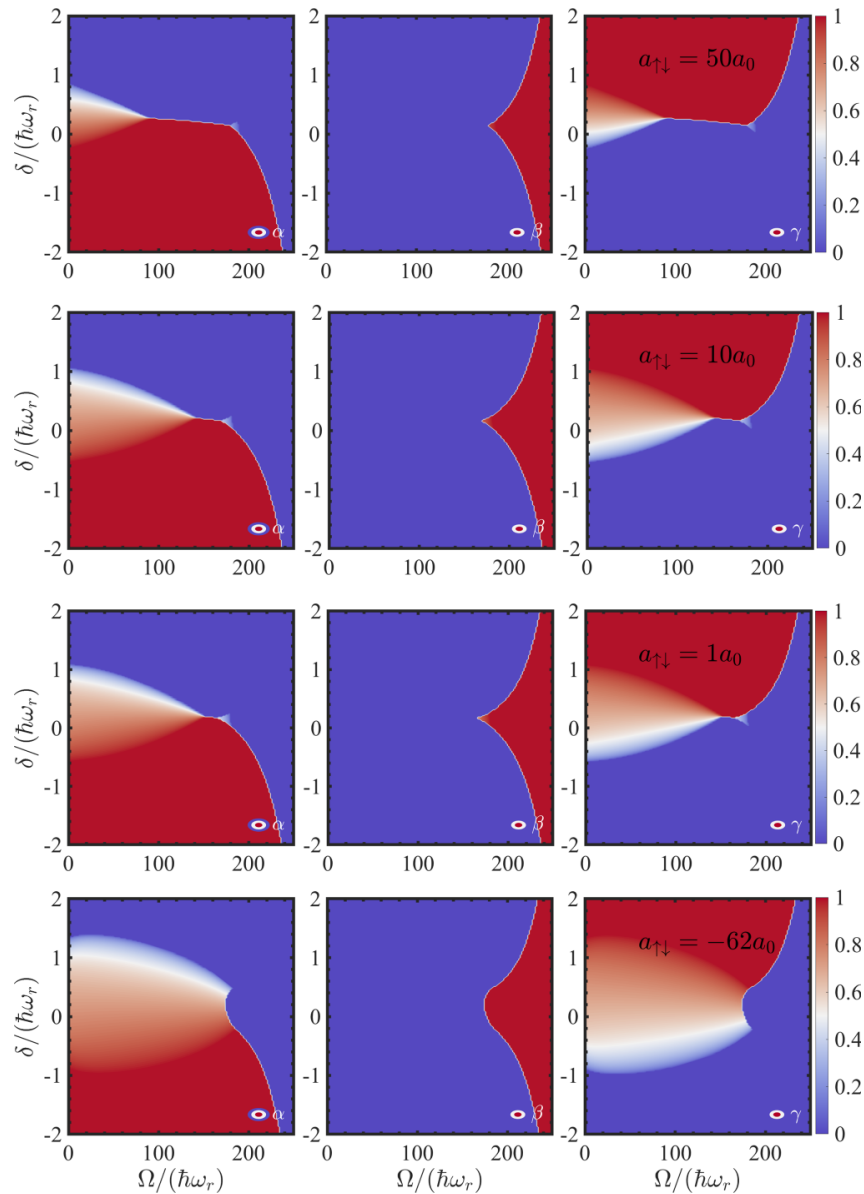

FIG. 9. Contour plots of the portions $(\alpha, \beta$, and $\gamma)$ of different $l_{z}$ states in the Ansatz for the condensate wave function from Eq. (9), in the $\delta$ - $\Omega$ plane. From top to bottom, the interspecies scattering length $a_{\uparrow \downarrow} / a_{0}$ is tuned to $50,10,1$, and -62 , respectively, via the Feshbach resonance in ${ }^{41} \mathrm{~K}$ atoms [40,41].

as $\boldsymbol{\Psi}(r, \phi)=\alpha e^{i \theta_{\alpha}} \psi_{-1}+\beta e^{i \theta_{\beta}} \psi_{0}+\gamma e^{i \theta_{\gamma}} \psi_{1}$, with three nonnegative weights $(\alpha, \beta$, and $\gamma)$ satisfying the normalization condition $\alpha^{2}+\beta^{2}+\gamma^{2}=1$ and with $\psi_{l_{2}}$ corresponding to the definite-angular-momentum state $\left|l_{z}=-1 \hbar, 0,1 \hbar\right\rangle$ obtained by self-consistently solving the Gross-Pitaevskii equation (6). Therefore, the phase diagram in the $\delta$ - $\Omega$ plane can be determined by calculating the values of the weights $\alpha, \beta$, and $\gamma$.

In this Appendix we take four sets of interspecies scattering length $a_{\uparrow \downarrow}=(50,10,1,-62) a_{0}$ and determine the respective weights $\alpha, \beta$, and $\gamma$, as depicted from top to bottom in Fig. 9 . The case with $a_{\uparrow \downarrow}=10 a_{0}$ is explicitly discussed in the main text. In general, all four generic phases I, II, III, and IV can be found in the plots (see also the labels in Fig. 5). It is straightforward to find that, as we decrease $a_{\uparrow \downarrow}$ from positive to negative, i.e., increasing the relative interaction difference $g-g_{\uparrow \downarrow}$, stripe phase III (denoted by the gradient color on the left-hand side) is significantly enlarged over the ranges of Rabi frequency $\Omega$ and laser detuning $\delta$, similar to what is found in the Raman SOC [8]. Below a critical value of $a_{\uparrow \downarrow}$, phases I and III will tend to encounter and squeeze out phase II over the range of $\Omega$ at $\delta \sim 0$ (see, for instance, the 
bottom row in Fig. 9). We have particular interest in angular stripe phase III, which seems to possess two relatively large parameter spaces in the $\delta$ - $\Omega$ plane, i.e., about $1 \hbar \omega_{r}-2 \hbar \omega_{r}$ and $100 \hbar \omega_{r}-200 \hbar \omega_{r}$, respectively. Here the unit of energy is the radial harmonic trapping frequency $\hbar \omega_{r}=2 \pi \times 10 \mathrm{~Hz}$. Thus the large windows of parameters $\delta$ and $\Omega$ could be approximately $60-120 \mathrm{~Hz}$ and $6-12 \mathrm{kHz}$, respectively, which could be easily accessible in the laboratory.
[1] J. Dalibard, F. Gerbier, G. Juzeliūnas, and P. Öhberg, Rev. Mod. Phys. 83, 1523 (2011).

[2] N. Goldman, G. Juzeliūnas, P. Öhberg, and I. B. Spielman, Rep. Prog. Phys. 77, 126401 (2014).

[3] V. Galitski and I. B. Spielman, Nature (London) 494, 49 (2013).

[4] H. Zhai, Rep. Prog. Phys. 78, 026001 (2015).

[5] C. Wang, C. Gao, C.-M. Jian, and H. Zhai, Phys. Rev. Lett. 105, 160403 (2010).

[6] W. Cong-Jun, I. Mondragon-Shem, and Z. Xiang-Fa, Chin. Phys. Lett. 28, 097102 (2011).

[7] T.-L. Ho and S. Zhang, Phys. Rev. Lett. 107, 150403 (2011).

[8] Y. Li, L. P. Pitaevskii, and S. Stringari, Phys. Rev. Lett. 108, 225301 (2012).

[9] M. Boninsegni and N. V. Prokof'ev, Rev. Mod. Phys. 84, 759 (2012).

[10] Y.-J. Lin, K. Jimenez-Garcia, and I. B. Spielman, Nature (London) 471, 83 (2011).

[11] S.-C. Ji, J.-Y. Zhang, L. Zhang, Z.-D. Du, W. Zheng, Y.-J. Deng, H. Zhai, S. Chen, and J.-W. Pan, Nat. Phys. 10, 314 (2014).

[12] G. I. Martone, Y. Li, and S. Stringari, Phys. Rev. A 90, 041604(R) (2014).

[13] X.-L. Chen, J. Wang, Y. Li, X.-J. Liu, and H. Hu, Phys. Rev. A 98, 013614 (2018).

[14] J.-R. Li, J. Lee, W. Huang, S. Burchesky, B. Shteynas, F. Ç. Top, A. O. Jamison, and W. Ketterle, Nature (London) 543, 91 (2017).

[15] Y.-X. Hu, C. Miniatura, and B. Grémaud, Phys. Rev. A 92 , 033615 (2015).

[16] M. DeMarco and H. Pu, Phys. Rev. A 91, 033630 (2015).

[17] C. Qu, K. Sun, and C. Zhang, Phys. Rev. A 91, 053630 (2015).

[18] K. Sun, C. Qu, and C. Zhang, Phys. Rev. A 91, 063627 (2015).

[19] L. Chen, H. Pu, and Y. Zhang, Phys. Rev. A 93, 013629 (2016).

[20] H.-R. Chen, K.-Y. Lin, P.-K. Chen, N.-C. Chiu, J.-B. Wang, C.A. Chen, P.-P. Huang, S.-K. Yip, Y. Kawaguchi, and Y.-J. Lin, Phys. Rev. Lett. 121, 113204 (2018).

[21] P.-K. Chen, L.-R. Liu, M.-J. Tsai, N.-C. Chiu, Y. Kawaguchi, S.-K. Yip, M.-S. Chang, and Y.-J. Lin, Phys. Rev. Lett. 121, 250401 (2018).
[22] D. Zhang, T. Gao, P. Zou, L. Kong, R. Li, X. Shen, X.-L. Chen, S.-G. Peng, M. Zhan, H. Pu, and K. Jiang, Phys. Rev. Lett. 122, 110402 (2019).

[23] V. N. Popov, Functional Integrals and Collective Excitations (Cambridge University Press, Cambridge, 1991).

[24] A. Griffin, Phys. Rev. B 53, 9341 (1996).

[25] H. Hu, B. Ramachandhran, H. Pu, and X.-J. Liu, Phys. Rev. Lett. 108, 010402 (2012).

[26] B. Ramachandhran, B. Opanchuk, X.-J. Liu, H. Pu, P. D. Drummond, and H. Hu, Phys. Rev. A 85, 023606 (2012).

[27] I. Vasić and A. Balaž, Phys. Rev. A 94, 033627 (2016).

[28] X.-L. Chen, X.-J. Liu, and H. Hu, Phys. Rev. A 96, 013625 (2017).

[29] S. Stock, Z. Hadzibabic, B. Battelier, M. Cheneau, and J. Dalibard, Phys. Rev. Lett. 95, 190403 (2005).

[30] P. Cladé, C. Ryu, A. Ramanathan, K. Helmerson, and W. D. Phillips, Phys. Rev. Lett. 102, 170401 (2009).

[31] J.-y. Choi, S. W. Seo, and Y.-i. Shin, Phys. Rev. Lett. 110, 175302 (2013).

[32] G. I. Martone, Y. Li, L. P. Pitaevskii, and S. Stringari, Phys. Rev. A 86, 063621 (2012).

[33] J.-Y. Zhang, S.-C. Ji, Z. Chen, L. Zhang, Z.-D. Du, B. Yan, G.-S. Pan, B. Zhao, Y.-J. Deng, H. Zhai, S. Chen, and J.-W. Pan, Phys. Rev. Lett. 109, 115301 (2012).

[34] M. A. Khamehchi, Y. Zhang, C. Hamner, T. Busch, and P. Engels, Phys. Rev. A 90, 063624 (2014).

[35] S.-C. Ji, L. Zhang, X.-T. Xu, Z. Wu, Y. Deng, S. Chen, and J.-W. Pan, Phys. Rev. Lett. 114, 105301 (2015).

[36] W. Zheng and Z. Li, Phys. Rev. A 85, 053607 (2012).

[37] Y. Li, G. I. Martone, and S. Stringari, Europhys. Lett. 99, 56008 (2012).

[38] W. Zheng, Z.-Q. Yu, X. Cui, and H. Zhai, J. Phys. B 46, 134007 (2013).

[39] L. P. Pitaevskii and A. Rosch, Phys. Rev. A 55, R853 (1997).

[40] M. Lysebo and L. Veseth, Phys. Rev. A 81, 032702 (2010).

[41] L. Tanzi, C. R. Cabrera, J. Sanz, P. Cheiney, M. Tomza, and L. Tarruell, Phys. Rev. A 98, 062712 (2018). 